Review Article

\title{
Heritability and Genetics of Type 2 Diabetes Mellitus in Sub- Saharan Africa: A Systematic Review and Meta-Analysis
}

\author{
Evans Adu Asamoah $(\mathbb{D}$, Christian Obirikorang $(\mathbb{D}$, Emmanuel Acheampong $(\mathbb{D}$, \\ Max Efui Annani-Akollor, Edwin Ferguson Laing, Eddie-Williams Owiredu $(\mathbb{D}$, \\ and Enoch Odame Anto
}

Department of Molecular Medicine, School of Medicine and Dentistry, Kwame Nkrumah University of Science and Technology, Ghana

Correspondence should be addressed to Emmanuel Acheampong; emmanuelachea1990@yahoo.com

Received 12 February 2020; Accepted 8 June 2020; Published 2 July 2020

Academic Editor: Bernd Stratmann

Copyright @ 2020 Evans Adu Asamoah et al. This is an open access article distributed under the Creative Commons Attribution License, which permits unrestricted use, distribution, and reproduction in any medium, provided the original work is properly cited.

\begin{abstract}
Objectives. Sub-Saharan Africa (SSA) is observing an accelerating prevalence rate of type 2 diabetes mellitus (T2DM) influenced by gene-environment interaction of modifiable and nonmodifiable factors. We conducted a systematic review and meta-analysis on the heritability and genetic risk of T2DM in SSA. Methods. We reviewed all published articles on T2DM in SSA between January 2000 and December 2019 and available in PubMed, Scopus, and Web of Science. Studies that reported on the genetics and/or heritability of T2DM or indicators of glycaemia were included. Data extracted included the study design, records of family history, pattern and characteristics of inheritance, genetic determinants, and effects estimates. Results. The pattern and characteristics of T2DM heritability in SSA are preference for maternal aggregation, higher among first degree compared to second-degree relatives; early age-onset ( $<50$ years), and inherited abnormalities of beta-cell function/mass. The overall prevalence of T2DM was $28.2 \%$ for the population with a positive family history (PFH) and $11.2 \%$ for the population with negative family history (NFH). The pooled odds ratio of the impact of PFH on T2DM was 3.29 (95\% CI: 2.40-4.52). Overall, 28 polymorphisms in 17 genes have been investigated in relation with T2DM in SSA. Almost all studies used the candidate gene approach with most $(45.8 \%)$ of genetic studies published between 2011 and 2015. Polymorphisms in ABCC8, Haptoglobin, KCNJ11, ACDC, ENPP1, TNF- $\alpha$, and TCF7L2 were found to be associated with T2DM, with overlapping effect on specific cardiometabolic traits. Genome-wide studies identified ancestry-specific signals (AGMO-rs73284431, VT11A-rs17746147, and ZRANB3) and TCF7L2-rs7903146 as the only transferable genetic risk variants to SSA population. TCF7L2-rs7903146 polymorphism was investigated in multiple studies with consistent effects and low-moderate statistical heterogeneity. Effect sizes were modestly strong [odds ratio $=6.17$ (95\% CI: 2.03-18.81), codominant model; 2.27 (95\% CI: 1.50-3.44), additive model; 1.75 (95\% CI: 1.18-2.59), recessive model]. Current evidence on the heritability and genetic markers of T2DM in SSA populations is limited and largely insufficient to reliably inform the genetic architecture of T2DM across SSA regions.
\end{abstract}

\section{Introduction}

Studies on chronic noncommunicable diseases (NCDs) have gained considerable attention in Africa. This is as a result of the dramatic shift in the profile of diseases burden with the dominance of communicable diseases to NCDs in SubSaharan Africa (SSA) [1]. Africa constitutes the highest proportion of people with undiagnosed diabetes $(>50 \%)$ and has been projected to experience further future increase $(>100 \%)$ in the burden of diabetes by 2045 [2]. Between the periods of 1990 and 2017, NCDs have accounted for a 67\% increase in disability-adjusted life-years (DALY) in SSA, and type- 2 diabetes mellitus (T2DM) accounted for $126 \%$ increase in DALY [3]. The regional prevalence of T2DM in SSA is reportedly $5.1 \%$ [4], which range from $2.6 \%$ to $22.5 \%$ across data from different regions in SSA [5]. Between 1990 and 2015 , T2DM has accounted for $7 \%$ mortality rate attributed to NCDs in SSA [6]. 
In Africa, ageing and globalisation including nutrition transition and adoption of sedentary lifestyles are reportedly key factors accelerating diabetes prevalence, in line with the worldwide rise in overweight and obesity $[7,8]$. The causal linkages of T2DM reported in most studies in Africa include environmental/behavioural cause, direct multifactorial cause, and indirect multifactorial cause, respectively. Direct multifactorial cause involves a network of risk factors directly causes T2DM overtime while indirect multifactorial cause is where aggregation of risk factors over a period cause a precursor health condition (obesity, hypertension, metabolic syndrome, etc.) that leads to T2DM [9]. Accumulated evidence from a number of studies confirms a gene-environment interaction of modifiable and nonmodifiable factors, which promote the development of diabetes [10-12]. However, a limitation to the understanding of the epidemiology of T2DM in SSA has been the lack of established vital statistics systems and reliable population-level data for most countries [13]. More unfavourably, in the face of recent implementations of high throughput genotyping and sequencing approaches to advance recent understanding of the genetic basis of T2DM, countries in SSA have not been adequately represented in the global genomic efforts [14]. Thus, studies to provide ample data in the areas of heritability, family history, and genetic risk of T2DM are warranted. As such, these studies will highlight new genetic initiatives towards the prevention and treatment of T2DM in SSA.

T2DM phenotype in SSA has been characterized by early onset in a younger population ( $<50$ years) $[15]$ and pancreatic beta-cell secretory dysfunction which manifests as the blunted acute first phase of insulin secretion rather than peripheral insulin resistance $[16,17]$. These characteristics of T2DM have been confirmed in the reports of Oli et al. [18] and Bakari and Onyemelukwe [19]. Thus, T2DM in SSA like the Asians [20], but unlike the Europeans [21], follows a different course that reflects environmental and genetics contribution to the high burden. Whereas the contribution of environmental factors to the development of T2DM in SSA is well established, the contribution of genetic factors remains elusive. There is currently no thorough systematic review and metaanalysis reporting on the genetics and heritability of T2DM in SSA. An up-to-date and accurate documentation on the genetics and heritability of T2DM in SSA is needed to highlight gaps and if applicable inform policymakers to design and implement public health intervention programs. This study recapitulates literature on the genetics and heritability of T2DM currently available in SSA.

\section{Methodology}

2.1. Search Strategy and Selection Criteria. Following the Preferred Reporting Items for Systematic Reviews and Meta-Analyses (PRISMA) guidelines [22] and the protocol for conducting a systematic review for genetic association studies [23], we conducted a systematic review of all papers published on T2DM in Sub-Saharan Africa between January 2000 and November 2019 and available on PubMed, Scopus, and Web of Science. We defined Sub-Saharan Africa as all mainland African countries south of the Sahara excluding Algeria, Djibouti, Egypt, Libya, Morocco, Somalia, Sudan, and Tunisia. Boolean logic with search terms including "type 2 diabetes", "non-insulin-dependent diabetes mellitus", Type-2 diabetes mellitus", and "Africa south of Sahara" were used. We applied the Medical Subject Heading terms (MeSH) to identify synonyms. We limited our search to studies done in English. The study protocol and detailed search parameters are attached to this manuscript as Supplementary Material (available here). The reference lists of study reports and review articles were scanned to identify additional articles validated in Science Citation Index Expanded, published on and after January 2000.

Two authors (EAA and EA) independently reviewed the title and abstracts of studies for inclusion. Cross-sectional studies (community-based, population-based, hospital-based, and comparative), case-control, and prospective cohort studies that reported a heritability, family history, and genetic risk of T2DM in SSA were eligible for inclusion. We regarded the following terms as equivalent to type-2 diabetes mellitus: "Type 2 Diabetes", "Diabetes Mellitus, Slow Onset", "Noninsulin-Dependent Diabetes Mellitus", "Maturity Onset Diabetes Mellitus", “Diabetes Mellitus, Stable”, "Diabetes Mellitus, Type II", “Adult-Onset Diabetes Mellitus”, "Diabetes Mellitus, Noninsulin Dependent", "NIDDM", "Diabetes, Maturity-Onset", "Diabetes, Type 2", "Slow-Onset Diabetes Mellitus", "Diabetes Mellitus, Non-Insulin-Dependent”, "Diabetes Mellitus, Ketosis-Resistant”, "Ketosis-Resistant Diabetes Mellitus", and "Maturity-Onset Diabetes Mellitus". Two investigators (EAA and EWO) did a second review to assess the entire articles and their reference list. Any disagreement resulted in a joint review of the article with reconciliation. Details of inclusion and exclusion criteria can be found in Supplementary Appendix, pp 4.

2.2. Data Extraction. After identifying articles for inclusion, two authors (EA and CO) independently reviewed each article for data extraction into a standard, preformulated form:

Family History of T2DM: data were extracted under the following headings: name of first author, publication year, Sub-Saharan Africa region, country, ethnicity, study design, participants, sampling technique, and sample size, criteria for T2DM definition, number of participants with a positive family history (PFH), T2DM prevalence in $\mathrm{PFH}$, and T2DM prevalence in negative family history $(\mathrm{NFH})$.

Heritability: themes were used to pool key concepts and interpreted within and across studies. Themes used included T2DM aggregation among families, onset of T2DM among probands, glucose tolerance, and effectiveness mechanisms among probands and control participants.

Genetic Risk: data were extracted under the headings-first author and publication year; the population; gene (s) and allelic variant (s); total sample size; the number of nucleotide genes in which they are found and their corresponding genotypes both in cases and controls, genotyping method; minor allele frequency.

2.3. Statistical Analysis. For the polymorphisms investigated in multiple studies, we derived the pooled estimates of their association with T2DM risk across studies using a random 
effect model meta-analysis, implemented using MedCalc Software for Windows, version 18.91 (https://www.medcalc .org/). Heterogeneityin the measure of association across studies was further quantified with the $I^{2}$ statistic, with value $<25 \%$ indicating low heterogeneity, $25-50 \%$ indicating moderate heterogeneity, $50-75 \%$ indicating high heterogeneity, and $>75 \%$ indicating extreme heterogeneity [24]. Publication bias was assessed using funnel plots [25].

\section{Results}

3.1. Literature Search. The search strategy and the number of hints per each search are shown in Supplementary Appendix pp 1-2. A total of 3,519 published records were identified through searches (Figure 1). A total of 718 studies were excluded based on duplication, and titles/abstracts of 2913 records were screened. Of these, 341 were included for fulltext review, and 4 additional studies were obtained from screening the references of included studies. A total of 44 studies consisting of $8 \mathrm{GWAS} /$ linkage studies, 4 pedigree/familial studies, 19 candidate gene association studies, and 13 population/hospital-based cross-sectional studies were included. Figure S1 shows the linear growth over time of heritability, family history, and genetic studies on T2DM published in SSA. Up to $45.8 \%$ of genetic studies were published between 2011 and 2015, and $66.7 \%$ of FH and heritability were published between 2011 and 2019.

3.2. Pedigree and Family Studies on T2DM in SSA. Although few published studies [26-29] have thoroughly investigated the heritability of T2DM within the SSA context, these studies demonstrate a distinct familial aggregation of T2DM and offered useful insights into the pattern of heritability of T2DM.

The reports of Meiloud et al. [26] among 609 known T2DM Mauritanian patients indicated that $27 \%$ of T2DM patients have at least one relative with the condition. The association between PFH and T2DM was observed to be higher among first-degree compared to second-degree relatives $(p=0.003)$. Moreover, more probands with an affected mother than those with affected father were observed, suggesting a preferential maternal effect which does not extend to second-degree relatives.

Similar to the above findings, a prospective case-control study among 1,111 T2DM and 687 controls from the Black South African population [27] reported 27.3\% of T2DM having a $\mathrm{PFH}$ compared with $8.4 \%$ in the control group $(p<0.01)$. Also, first-degree relatives with T2DM had a significant maternal aggregation of $64.7 \%$ compared with $27 \%$ of those who had diabetic fathers $(p<0.01)$. In addition to these, Erasmus et al. [27] reported that patients with $\mathrm{PFH}$ had an earlier onset ( $<50$ years) of diabetes than those with NFH $(p<0.01)$.

Two of the studies included in this review reported severe pancreatic beta-cell secretory dysfunction among Ghanaian and Cameroonian offspring of T2DM patients compared with those of nondiabetic parents [28, 29]. Amoah et al. [29] compared $\beta$-cell secretion, insulin secretion, insulin sensitivity ( $\mathrm{Si}$ ), and glucose effectiveness $(\mathrm{Sg})$ among 42 healthy nondiabetic first-degree relatives and 22 healthy control subjects without a PFH The mean total and acute first and second phases of serum insulin and c-peptide responses after oral glucose tolerance test at $t=60,90$, and 120 minutes $(p<0.05)$ were prolonged in relatives than in healthy controls. The level of insulin resistance and glucose effectiveness at basal insulin level (Sg) did not significantly differ in the relatives and healthy controls [29].

Even though only few studies $(<5)$ have investigated the familial aggregation of T2DM in SSA, three common themes were identified to be associated with T2DM heritability. Firstly, preference for maternal aggregation was higher among first degree compared to second-degree relatives; secondly, probands manifested a degree of $\beta$-cell impairment marked by reduced early-phase insulin secretion; and lastly, T2DM onset was early ( $<50$ years) among relatives of diabetic individuals.

3.3. Genome-Wide Association/Linkage Studies. The two maiden genome-wide linkage study in SSA among the Akan and Ga, from Ghana, Yoruba, and Igbo from Nigeria identified significant linkage signal regions on $10 \mathrm{q} 23$ and $4 \mathrm{p} 15$, while few other regions had logarithm odd (LOD) scores considered as suggestive evidence of linkage [30,31].

Using an affected sibling pair (ASP) approach with 390 short tandem repeat markers typed in 343 families in the African American Diabetes Mellitus (AADM) study, a multipoint linkage analysis identified suggestive evidence of linkage in four regions on three chromosomes; $12(\mathrm{LOD}=1.92), 19$ $(\mathrm{LOD}=1.81)$, and $20(\mathrm{LOD}=2.63)$, with the strongest evidence on 20q13.3 (LOD $=1.80)$. This region was previously identified as a T2DM susceptible locus in a nonAfrican population [32,33]. In addition, 12q24 linkage signal was identified, as has been previously reported [34] as T2DM susceptible locus.

Chen et al. [31] employed the multipoint linkage approach performed on $\log$ C-peptide using the AADM samples. Significant linkage signals were observed on 10q23 $(\mathrm{LOD}=4.04), 4 \mathrm{p} 15(\mathrm{LOD}=3.48), 15 \mathrm{q} 14(\mathrm{LOD}=2.41)$, and $18 \mathrm{p} 11(\mathrm{LOD}=2.18)$. These regions were identified to harbour five positional candidate genes for T2DM and related complications: the pituitary adenylate cyclase-activating polypeptide $(P A C A P)$ in 18p11; the peroxisome proliferatoractivated receptor-gamma coactivator 1 (PPARGC1) in 4p15; PTEN, PPP1R5, and IDE in 10q23 (Supplementary Appendix, pp.16).

Adeyemo et al. [35] conducted a study on the evaluation of T2DM susceptibility genome-wide loci in SSA. The study sampled 1775 subjects of which $90 \%$ were participants enrolled in the AADM study and $10 \%$ of East Africans enrolled from Kenya. The study observed 41 loci showing transferability to African sample: 11 at the exact reported SNP and 30 others at SNPs in linkage disequilibrium (LD) with the reported SNP. The TCF7L2 SNP rs7903146 showed the strongest association with T2DM $\left(p=1.61 \times 10^{-8}\right.$, OR $1.50,95 \%$ CI 1.26-2.15) [35]. When the 41 loci that showed transferability were fine-mapped, stronger evidence of association with T2DM was shown by neighbouring SNPs (SLC30A8 and CDKAL1) compared with index SNPs with 


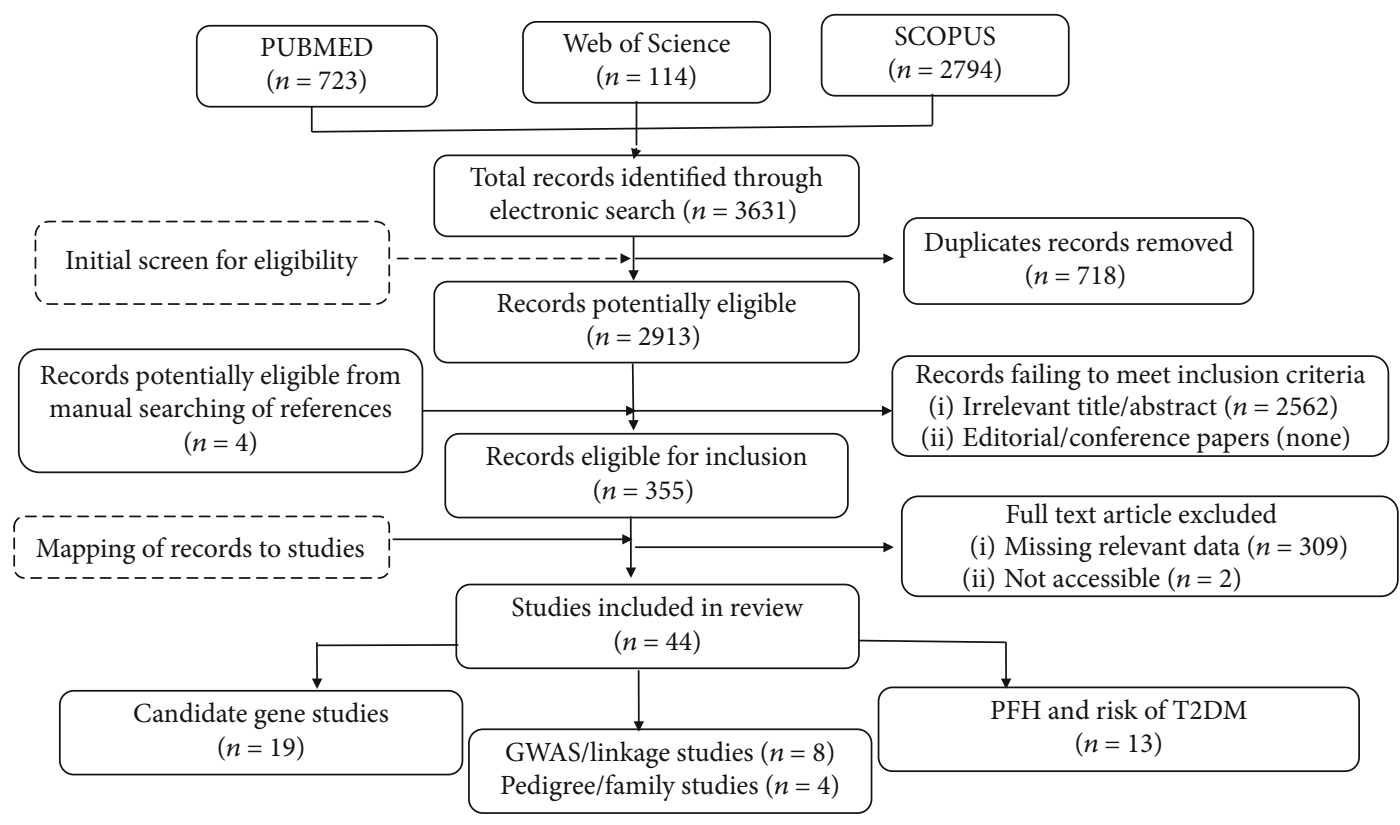

Figure 1: Flowchart for the study selection process.

an exception to the block that contained both TCF7L2 and ZBED3.

In a recent discovery study by Adeyemo et al. [36] using the population group of West and East Africans [35], ZRANB3 (lead SNP $p=2.831 \times 10^{-9}$ ), which has not been previously associated with T2DM, was identified as an African-specific T2DM locus. The role of ZRANB3 in an experimental model was found to be involved in $\beta$-cell functional response to high glucose conditions, thus the capacity of the pancreas to respond to insulinogenic stimuli [35].

A study by Chen et al. [37] found an association with T2DM using the shared variant (rs7903146) from West, East, and South Africa, the Europeans, and Africans and a distinct African-specific signal, VT11A-rs17746147. Likewise, one novel signal, rs73284431, near alkylglycerol monooxygenase ( $\left.A G M O: p=5.2 \times 10^{-9}, \mathrm{MAF}=0.095\right)$, distinct from previously reported signals in the African region was also detected. This indicates that combining different populations from SSA could possibly lead to the discovery of new and population-specific loci for T2DM.

3.4. Association between PFH and the Risk of T2DM among SSA Population. Thirteen studies were identified that provided evidence on the risk of T2DM among the general population. Ten of the studies were population/community-based cross-sectional studies [38-47], two were hospital-based case-control studies $[48,49]$, and one study was a comparative cross-sectional study [50]. A total of 14,432 participants from the ten cross-sectional studies and the two hospital-based case-control studies were used for meta-analysis. The characteristics of the 12 studies are shown in Table S4 (Supplementary Appendix pp. 10). From the pooled studies, the prevalence of T2DM ranged from 5.4$66.8 \%$ among participants with a positive family history (PFH) and 2.5-32.2\% among participants with no family history (NFH). The overall prevalence of T2DM among the
SSA population with PFH of T2DM was $28.2 \%$ compared with $11.2 \%$ among participants with NFH (Table 1).

A meta-analysis from the twelve studies demonstrated a significant effect of $\mathrm{PFH}$ on T2DM prevalence in SSA [OR = 3.29 (95\% CI: 2.40-4.52)] (Figure 2). This effect size was robust in the sensitivity analysis and remained at a significant level following the omission of each single study. A leave-one-out sensitivity analysis also revealed that the pooled estimate was much less impacted by odds ratios values from Bello-Ovosi et al. [40], Millogo et al. [44], and Mayega et al. [46] (Figure 3). The funnel plot revealed publication bias, depicted by the asymmetrical display of odds ratios reported by the various studies (Figure 4 ).

3.5. Candidate Gene Studies. A total of 28 polymorphisms (including SNP, indels, and repeats) in 17 genes were investigated across studies. Supplementary Document: pp. 6-10 shows the distribution of the polymorphisms in various genes, including four in CAPN10 (rs3792267, rs2975762, rs5030952, rs3842570), three in TCF7L2 (rs7903146; rs12255372; DG10S478), and FTO (rs9941349, rs3751812, rs8050136); and two in $A C D C$ (C-11377G, G-11391A) and ENPP1 (rs997509, rs1044498).

A shown in Supplementary Table S3, 8 SNPs in the SCL40A1, PPAR- $\gamma$, IRS-1, AGRP, PPAR- $\alpha$, and CAPN10 genes were examined and were not significantly associated with T2DM or measures of glycaemia across included studies. In single studies, the $A B C C 8$ and Haptoglobin SNPs were found to be associated with T2DM among Nigerians [51] and Ghanaians [52], respectively. Two polymorphisms in two genes (PSMD6 and C2D4B) were found to be nonsignificantly associated with T2DM in Black South Africans after correcting for multiple testing [53]. Polymorphisms in KCNJ11 ( $r$ 55219) was found to be nonsignificantly associated with T2DM among Ghanaian [49] and Nigerian [54] populations but significant among Mauritanians [55]. 
TABLE 1: shows the prevalence of T2DM among participants with a positive family history of T2DM.

\begin{tabular}{|c|c|c|c|c|c|}
\hline \multirow{2}{*}{ Study } & \multirow{2}{*}{ Sample size } & \multicolumn{2}{|c|}{ PFH population } & \multicolumn{2}{|c|}{ NFH population } \\
\hline & & Prevalence (\%) & $95 \% \mathrm{CI}$ & Prevalence (\%) & $95 \% \mathrm{CI}$ \\
\hline Bantie et al. [38] & 607 & 19.2 & $11.2-29.7$ & 8.9 & $6.6-11.6$ \\
\hline Zenebe et al. [39] & 264 & 35.1 & $20.2-52.5$ & 15.9 & $11.4-21.3$ \\
\hline Bello-Ovosi et al. [40] & 172 & 35.5 & $19.2-54.6$ & 22.0 & $15.5-29.7$ \\
\hline Wondemagegn et al. [41] & 714 & 23.1 & $17.0-30.1$ & 8.1 & $5.9-10.7$ \\
\hline Tesfaye et al. [42] & 851 & 19.6 & $10.2-32.4$ & 4.2 & $2.9-5.8$ \\
\hline Vuvor et al. [43] & 597 & 14.5 & $7.2-25.0$ & 2.5 & $1.3-4.2$ \\
\hline Millogo et al. [44] & 4415 & 5.4 & $2.7-9.4$ & 5.5 & $4.8-6.2$ \\
\hline Abebe et al. [45] & 2136 & 17.9 & $10.2-28.3$ & 3.1 & $2.4-3.9$ \\
\hline Mayega et al. [46] & 1497 & 20.9 & $15.2-27.5$ & 15.1 & $13.2-17.2$ \\
\hline Nyenwe et al. [47] & 492 & 26.3 & $13.4-43.1$ & 5.3 & $3.4-7.8$ \\
\hline Frank et al. [48] & 1221 & 65.0 & $60.54-69.19$ & 30.7 & $27.37-34.18$ \\
\hline Danquah et al. [49] & 1466 & 66.8 & $62.86-70.65$ & 32.2 & 29.16-35.44 \\
\hline Total (random) & 14,432 & 28.2 & $14.52-44.27$ & 11.2 & $6.39-17.06$ \\
\hline$I^{2}($ inconsistency $)=98.11$ & 0001 & & & & \\
\hline
\end{tabular}

PFH: positive family history; NFH: negative family history.

\begin{tabular}{lcccc}
\hline Study & Odds ratio & $95 \%$ CI & Weight (\%) \\
\hline Bantie et al., [36] & 2.44 & 1.29 to 4.62 & 8.05 \\
Zenebe et al., [37] & 2.87 & 1.34 to 6.16 & 7.05 \\
Bello-Ovosi et al., [38] & 1.95 & 0.84 to 4.51 & 6.51 \\
Wondemagegn et al., [39] & 3.42 & 2.13 to 5.48 & 9.45 \\
Tesfaye et al., [40] & 5.64 & 2.68 to 11.90 & 7.18 \\
Vuvor et al., [41] & 6.71 & 2.82 to 15.98 & 6.3 \\
Millogo et al., [42] & 0.98 & 0.53 to 1.83 & 8.18 \\
Abebe et al., [43] & 6.93 & 3.69 to 13.01 & 8.11 \\
Mayega et al., [44] & 1.48 & 1.00 to 2.18 & 10.14 \\
Nyenwe et al., [45] & 6.40 & 2.79 to 14.69 & 6.55 \\
Frank et al., [46] & 4.19 & 3.28 to 5.34 & 11.18 \\
Danquah et al., [47] & 4.24 & 3.39 to 5.29 & 11.3 \\
Total (random effects) & $\mathbf{3 . 2 9}$ & $\mathbf{2 . 4 0}$ to 4.51 & $\mathbf{1 0 0}$ \\
Heterogeneity: Chi-square = 53.63, df=11, $p<0.0001, I^{2}=79.49 \%$ \\
Test for overall effect: $z=7.38, p<0.001$
\end{tabular}

FIGURE 2: Forest plots for meta-analysis of family history and the risk of T2DM among SSA population.

Of the three polymorphisms in the FTO gene reported in two studies in two black South African mixed ancestry population, only $r s 9941349(\mathrm{OR}=1.43(1.00-2.04), p=0.052)$ was near significance, associated with T2DM after adjustment for cofounders [56].

With the two SNPs in ACDC reported among Black South Africans, SNP G-11391-A was found to be protective to T2DM [57]. Of the two SNPs in ENPP1 reported among South-African Mixed ancestry, only rs997509 was found to be significantly associated with T2DM [56]. Also, one SNP in TNF- $\alpha(-308 \mathrm{G} / \mathrm{A})$ was reported to be associated with T2DM among the Ethiopian population [58].
Moreover, all the variants of TCF7L2 reported among Ghanaians [59], Cameroonians [60, 61], and Nigerians [62] were found to be significantly associated with T2DM. Among South Africa-Mixed Ancestry, TCF7L2-rs7903146 showed marginal significance whereas TCF7L2-rs12255372 was not significantly associated with T2DM [56].

3.6. Association between TCF7L2-rs7903146 Risk Variant and T2DM among SSA Population. The pooled estimate of the effect of the TCF7L2-rs7903146 polymorphism on T2DM is shown in Figure 5. Across the three studies that assessed the effect of TCF7L2-rs7903146 using the codominant genetic 


\begin{tabular}{|c|c|c|c|}
\hline \multicolumn{3}{|c|}{ Statistics with study removed } & \multirow[b]{2}{*}{ Weight (\%) } \\
\hline Study & Odds ratio & $95 \% \mathrm{CI}$ & \\
\hline Vuvor et al., [41] & 6.71 & 2.82 to 15.98 & 3.61 \\
\hline Abebe et al., [43] & 6.93 & 3.69 to 13.01 & 6.52 \\
\hline Nyenwe et al., [45] & 6.40 & 2.79 to 14.69 & 3.91 \\
\hline Bantie et al., [36] & 2.44 & 1.29 to 4.62 & 6.39 \\
\hline Zenebe et al., [37] & 2.87 & 1.34 to 6.16 & 4.59 \\
\hline Wondemagegn et al., [39] & 3.42 & 2.13 to 5.48 & 10.81 \\
\hline Tesfaye et al., [40] & 5.64 & 2.68 to 11.90 & 4.79 \\
\hline Danquah et al., [47] & 4.24 & 3.39 to 5.29 & 31.17 \\
\hline Frank et al., [46] & 4.19 & 3.28 to 5.34 & 28.21 \\
\hline Total (fixed effects) & 4.15 & 3.61 to 4.77 & 100 \\
\hline \multicolumn{4}{|c|}{$\begin{array}{l}\text { Heterogeneity: Chi-square }=9.65, \mathrm{df}=8, p=0.290, I^{2}=17.11 \% \\
\text { Test for overall effect: } z=16.70, p<0.001\end{array}$} \\
\hline
\end{tabular}

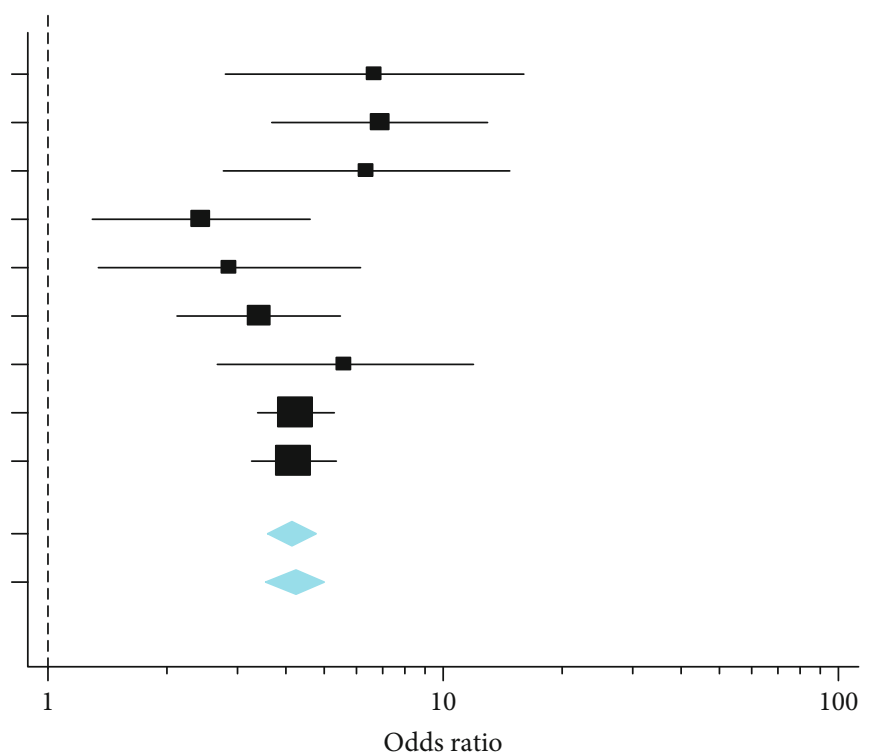

Figure 3: Leave-one-out sensitivity analysis of the effect of PFH on the risk of T2DM.

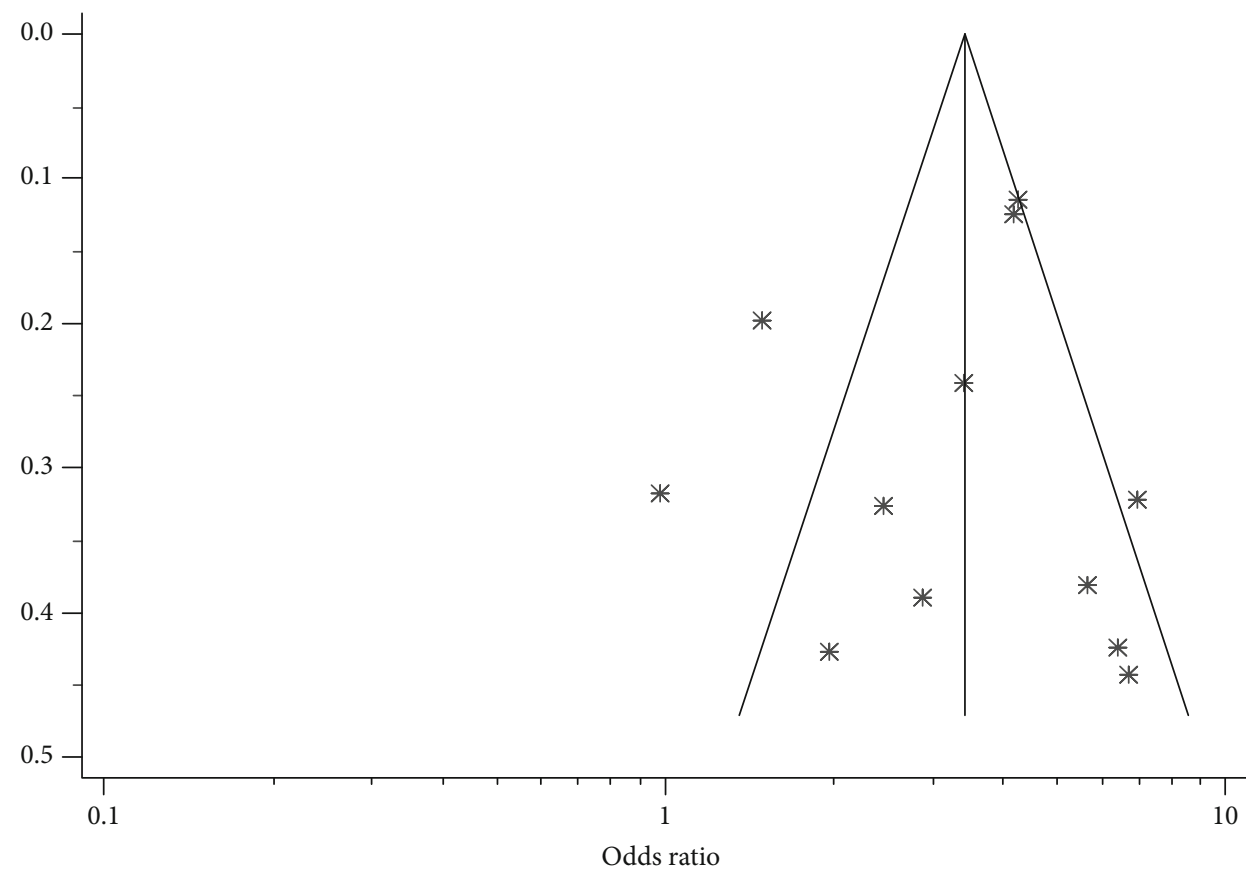

FIgURE 4: Funnel plots of studies reporting on family history and the risk of T2DM in SSA.

model, the pooled odds ratio was 6.17 (95\% CI: 2.03$18.81 ; I^{2}=64.71 \%, p$ - heterogeneity $\left.=0.059\right)$. The effect of TCF7L2-rs7903146 was 1.75 (95\% CI: $1.18-2.59 ; I^{2}=$ $0.00 \%, \mathrm{p}$ - heterogeneity $=0.859)$ using the recessive model across two studies; and 2.27 (95\% CI: 1.50-3.44; $I^{2}=0.00 \%$, $\mathrm{p}$ - heterogeneity $=0.789$ ) additive model across two studies. These values suggest that the effect of TCF7L2-rs7903146 on T2DM risk is moderately strong among the SSA population with moderate heterogeneity existing across studies.

\section{Discussion}

4.1. Pattern and Characteristics of T2DM Heritability in SSA. This review process provided a comprehensive and integrated perspective on heritability and genetic risk of T2DM in SSA. T2DM aggregation among the SSA population with $\mathrm{PFH}$ is 2.5 times the rate observed among the general population (28.2\% vs. $11.2 \%)$. Likewise, the risk of T2DM increases to about 3.3-fold among the population with $\mathrm{PFH}$ 


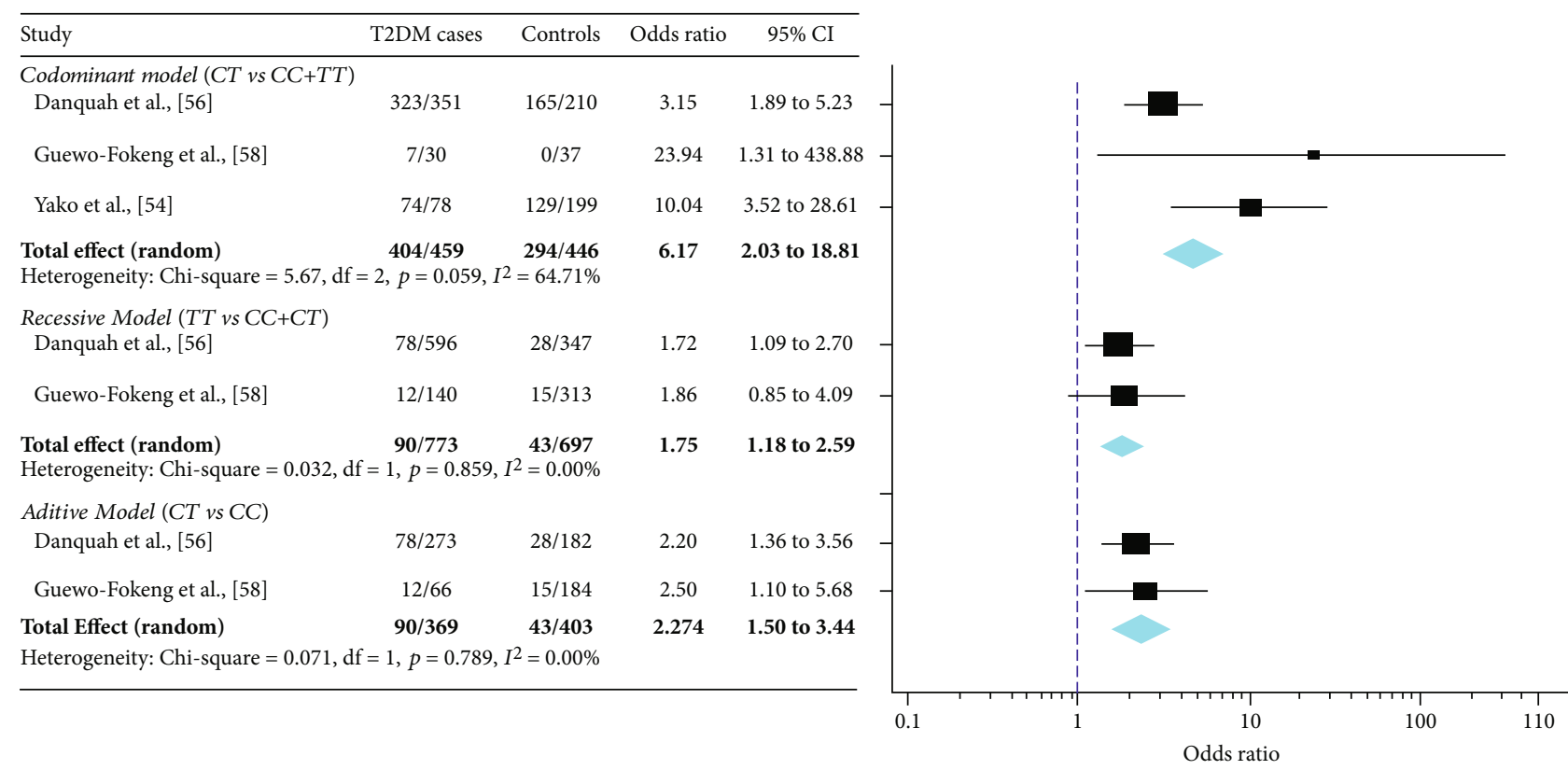

FIGURE 5: Forest plots for meta-analysis of the association between TCF7L2-rs7903146 and the risk of T2DM among the SSA population.

compared with those without a family history of T2DM. Notwithstanding, significant heterogeneity existed among studies. Evidence from a variety of population, family, and twin-based studies $[63,64]$ has provided an estimated range for the heritability of T2DM to be $20 \%-80 \%$, which is similar to that observed among SSA population. Another findings of Florez et al. [65] generally indicated that the risk of T2DM among the relative of individuals with T2DM is 3 times higher compared with individuals without a PFH. Thus, family history is not only an important risk factor for T2DM but also explains the importance of inheritance to the growing burden of T2DM among the population of SSA. It also represents a major role for inborn susceptibility to T2DM.

The features of T2DM heritability in SSA are of the preference of maternal to paternal aggregation and higher among first-degree relatives. These evidences have been confirmed by several studies across different population groups [26, 66-68]. However, some studies have reported no parental differences in disease transmission [63, 69, 70]. Earlier evidence suggested that considering the pattern of familial aggregation of $\mathrm{T} 2 \mathrm{DM}$, the possible explanations are true genetic maternal autosomal inheritance, which results from the passing on to offspring, maternally derived genetic variants in preference to paternal genes and/or maternal genes preferentially switched on during ontogeny [66, 71]. Based on current research evidence, it is possible that excess maternal influence may be explained by the intrauterine environment of the diabetic mother that may leave epigenetic signatures, which alter gene expressions responsible for insulin secretion, beta-cell and glucose uptake, and tolerance mechanisms [64]. Pettitt et al. [72] demonstrated that diabetic risk is high among the offspring of Pima Indian women who were diabetic during pregnancy than if the mothers develop diabetes later in life. In the present review, data were not available on the diabetic state of mothers at the time of pregnancy. Accordingly, a long-term prospective population study would be required to obtain these data with certainty, following up the offspring for the development of diabetes.

T2DM risk among the population with PFH acts through impaired insulin secretion as a result of beta-cell dysfunction rather than insulin action. Accordingly, genetic risk variants identified as ancestry-specific signals through fine mapping of causal variants (AGMO-rs73284431, VT11A-rs17746147, and ZRANB3), and the transferable genetic risk variants (TCF7L2), are reported to act through beta-cell dysfunction to confer the risk of T2DM $[36,37]$. Moreover, linkage signals detected among the SSA population from the two maiden linkage studies $[30,31]$ were loci that contain genes involved in beta-cell function instead of insulin action. This establishes that inherited abnormalities of beta-cell function/mass are critical precursors of T2DM among the SSA population. Typical of T2DM phenotype in SSA, Kibirige et al. [73] in a narrative review indicated that hyperglycaemia in most cases are predominantly characterised by delayed acute first phase of insulin secretion and pancreatic beta-cell secretory dysfunction, rather than peripheral insulin resistance.

The inheritance basis of T2DM among the SSA population, like many other groups, is typical of multifactorial inheritance of several different genes and likely epi-alleles that affects beta-cell function [74]. In examining T2DM in SSA, Kibirige et al. [73] reported that chronic inflammation, early life malnutrition, and epigenetic modifications are potential contributors to the distinct differences in disease manifestation. It is also true that insulin resistance is a precursor for T2DM, and insulin activity may be subject to genetic variance at several loci. Thus, to understanding the details of the pattern and characteristics of T2DM heritability in SSA, detailed studies with the focus on the genetic characteristics of familial T2DM pedigrees, glucose transport and tolerance mechanisms, as well as insulin secretion mechanisms among T2DM probands (considering the effect of 
biological ageing and environmental stressors on the expression of insulin signalling proteins) will be useful.

4.2. Genetics Risk of T2DM in SSA. Existing studies in SSA have tested 27 polymorphisms in 16 genes that have been reported to be associated with T2DM in other population groups. The candidate gene study approach was used with trends mostly toward consistent effect for genes replicated in multiple studies and modest effect size of the variant with a positive signal. Regarding the effect of the European common variant (TCF7L2) and Asian common variant KCNQ) with the greatest effect, only TCFL2 has been replicated among the SSA population and its role defined. Genomewide association/linkage studies revealed linkage signals in chromosome regions harbouring these two genes; however, studies of selected SNPs found an almost consistent association of TCF7L2 with T2DM whereas that of KCNQ was not replicated.

A meta-analysis of GWAS has consistently reported that some T2DM genetic risk signals are transferable across populations, whereas others are population-specific $[75,76]$.

We identified three studies that have explored the human genome using GWAS in the SSA population and uncovered evidence for three ancestry-specific signals (AGMO, VT11A, and ZRANB3), which are distinct for African population [35-37]. In addition, TCF7L2 was identified to be transferable to populations within SSA. Heterogeneity studies of the effect of transferable genetic risk variants across the population have been consistent with the reporting of the effect estimate. Similarly, the effect estimate of TCF7L2 among the SSA population was within the range reported among Asians and Europeans. Thus, there is the homogeneity of the effect of TCF7L2 variants across the population, which is likely to project a vital contribution of differences in environmental factors to the T2DM prevalent rate.

Whether or not, and to what extent GWAS signals identified in other non-African populations would be transferable across SSA populations remains a question to be answered. Besides the evidence of the transferability of TCF7L2 among the SSA population, others like KCNJ11 (rs5219) were among Mauritanians [55] but not Ghanaian [49] and Nigerian [54] populations. This has also been generally observed among the entire African population [77]. The inconsistencies are likely due to factors including differences in underlying genetic architecture, inadequate sample size, and genotyping methods. Further contributing to these inconsistencies, there exist a report of exaggerated effect estimates that is often seen in the earliest association studies compared with subsequent attempts to replicate the finding [78]. Overall, systematic approaches that take into consideration these limitations are needed to adequately answer the question of how well loci identified in non-Africa populations transfer across African populations. These systematic approaches have been applied in a few studies [35-37] and unravelled both global cosmopolitan and African specific alleles in T2DM aetiology.

4.3. Research and Clinical Perspectives on Heritability and Genetic Risk of T2DM in SSA. All the SNPs identified or replicated in SSA are within introns or intergenic regions.
Accordingly, their functional significance remains to be resolved, and evidence suggests their involvement in the regulation of gene expression or splicing [79]. SNPs associated with common traits by far have been found to be enriched for expression quantitative trait loci (eQTLs) [80]. Hence, among the SSA population, additional insights on the genetic architecture of T2DM can be explored through transcriptomics, quantification, and expression profiling of genes in specific tissues using microarrays and high throughput RNA sequencing technologies (RNAseq).

One important question to consider among the SSA population is how well do the genetic and environmental factors interacts for T2DM expression and inheritance? Illustratively, evidence from nongenetic factors establishes a direct relationship between T2DM and insulin resistance, whereas heritability also presents a characteristic T2DM arising from beta-cell dysfunction. Therefore, genetic and nongenetic forms of T2DM can be characterized in SSA, where the disease can occur either in isolation or with affected children born to unaffected parents. If there is more to this, detailed analysis of epigenetic mechanisms in T2DM development in a larger sample size merits attention among SSA populations. Furthermore, the wide genetic diversity of the African population and differences in the distribution of nongenetic factors present researchers with opportunities to investigate gene-environment interaction (epigenetic) questions.

Matsha et al. [81] in a study among the Black South African population indicated that epigenetic changes are likely to be an early process that occurs before the onset of overt diabetes. Also, in the first Epigenome-wide Association Study in SSA, four novel differentially methylated probes (DMPs) at epigenome-wide level were identified, which provided insights into the epigenetic loci that underlie the burden of T2DM [82]. Demonstratively, palmitate exposure to human pancreatic islets induced global and specific DNA methylation alterations that resulted in coordinated changes in mRNA expression and decreased insulin secretion [83]. Therefore, better characterization of nongenetic risk factors and investigation of their synergistic effects with putative genetic risk variants will be needed for the proper tailoring of clinical and public health intervention strategies of T2DM in SSA.

\section{Conclusion}

This is the first meta-analysis to investigate the heritability and genetic risk of T2DM among SSA population. The current evidence on the heritability and genetic markers of T2DM in SSA populations is limited and largely insufficient to reliably inform the genetic architecture of T2DM across SSA regions and to inform clinical management of T2DM. Thus, studies to provide ample data in the areas of heritability, family history, and genetic risk of T2DM are warranted.

\section{Conflicts of Interest}

The authors declare that they have no conflicts of interest. 


\section{Authors' Contributions}

$\mathrm{CO}$ and MAA designed the study. EOA and EWO worked on data collection. EAA and EA analysed the data, interpreted the results, and wrote the paper. All authors reviewed and revised the manuscript and approved the manuscript before submission.

\section{Supplementary Materials}

Supplementary Appendix 1: methodological details and characteristics of included studies (Supplementary Materials). (Supplementary Materials)

\section{References}

[1] Organization WH, Political declaration of the high-level meeting of the general assembly on the prevention and control of non-communicable diseases. 66th Session of the Unites Nations General Assembly, WHO, New York, 2011.

[2] IDF, IDF Diabetes Atlas 8th Edition 2017, International Diabetes Federation, 2017, ISBN: 978-2-930229-87-4.

[3] H. N. Gouda, F. Charlson, K. Sorsdahl et al., "Burden of noncommunicable diseases in sub-Saharan Africa, 1990-2017: results from the Global Burden of Disease Study 2017," The Lancet Global Health, vol. 7, no. 10, pp. e1375-e1387, 2019.

[4] M. J. Nyirenda, "Non-communicable diseases in sub-Saharan Africa: understanding the drivers of the epidemic to inform intervention strategies," International health, vol. 8, no. 3, pp. 157-158, 2016.

[5] Organization WH, Report on the status of major health risk factors for noncommunicable diseases, WHO African Region, 2015.

[6] Collaborators GBDRF, "Global, regional, and national comparative risk assessment of 79 behavioural, environmental and occupational, and metabolic risks or clusters of risks, 1990-2015: a systematic analysis for the Global Burden of Disease Study 2015," Lancet, vol. 388, no. 10053, pp. 1659-1724, 2016.

[7] A. A. Motala, M. A. K. Omar, and F. J. Pirie, "Epidemiology of type 1 and type 2 diabetes in Africa," Journal of cardiovascular risk, vol. 10, no. 2, pp. 77-83, 2003.

[8] N. S. Levitt, "Diabetes in Africa: epidemiology, management and healthcare challenges," Heart, vol. 94, no. 11, pp. 13761382, 2008.

[9] A. Issaka, Y. Paradies, and C. Stevenson, "Modifiable and emerging risk factors for type 2 diabetes in Africa: a systematic review and meta-analysis protocol," Systematic Reviews, vol. 7, no. 1, p. 139, 2018.

[10] F. B. Hu, "Globalization of diabetes: the role of diet, lifestyle, and genes," Diabetes Care, vol. 34, no. 6, pp. 1249-1257, 2011.

[11] K. G. M. M. Alberti, P. Zimmet, and J. Shaw, "International Diabetes Federation: a consensus on type 2 diabetes prevention," Diabetic Medicine., vol. 24, no. 5, pp. 451-463, 2007.

[12] D. Noble, R. Mathur, T. Dent, C. Meads, and T. Greenhalgh, "Risk models and scores for type 2 diabetes: systematic review," BMJ, vol. 343, no. nov28 1, p. d7163, 2011.

[13] S. Dalal, J. J. Beunza, J. Volmink et al., "Non-communicable diseases in sub-Saharan Africa: what we know now," International journal of epidemiology, vol. 40, no. 4, pp. 885-901, 2011.
[14] M. C. Y. Ng, "Genetics of type 2 diabetes in African Americans," Curr Diab Rep, vol. 15, no. 10, p. 74, 2015.

[15] G. V. Gill, A. Tekle, A. Reja et al., "Immunological and Cpeptide studies of patients with diabetes in northern Ethiopia: existence of an unusual subgroup possibly related to malnutrition," Diabetologia, vol. 54, no. 1, pp. 51-57, 2011.

[16] A. Amoah, S. K. Owusu, D. P. Schuster, and K. Osei, "Pathogenic mechanism of type 2 diabetes in Ghanaians-the importance of beta cell secretion, insulin sensitivity and glucose effectiveness," Suid-Afrikaanse tydskrif vir geneeskunde, vol. 92, no. 5, pp. 377-384, 2002.

[17] A. G. Amoah, D. P. Schuster, T. Gaillard, and K. Osei, "Insulin resistance, beta cell function and cardiovascular risk factors in Ghanaians with varying degrees of glucose tolerance," Ethnicity and Disease, vol. 12, no. 4, pp. S3-10, 2002.

[18] J. M. Oli, A. A. Adeyemo, G. O. Okafor et al., "Basal insulin resistance and secretion in Nigerians with type 2 diabetes mellitus," Metabolic syndrome and related disorders, vol. 7, no. 6, pp. 595-600, 2009.

[19] A. G. Bakari and G. C. Onyemelukwe, "Insulin resistance in type 2 diabetic Nigerians," International Journal of Diabetes and Metabolism, vol. 13, no. 1, p. 24, 2019.

[20] Y. M. Cho, "Characteristics of the pathophysiology of type 2 diabetes in Asians," ANNALS OF LAPAROSCOPIC AND ENDOSCOPIC SURGERY, vol. 2, 2017.

[21] Z. Jakab, Delivering for diabetes in Europe. Plenary Meeting of the European Parliament's EU, Diabetes Working Group, held under the auspices of the Belgian Presidency of the EU; 8th December 2010, World Health Organization, 2010.

[22] A. Liberati, D. G. Altman, J. Tetzlaff et al., "The PRISMA statement for reporting systematic reviews and meta-analyses of studies that evaluate health care interventions: explanation and elaboration," PLoS medicine, vol. 6, no. 7, p. e1000100, 2009.

[23] G. S. Sagoo, J. Little, and J. P. T. Higgins, "Systematic reviews of genetic association studies," PLOS Medicine., vol. 6, no. 3, p. e28, 2009.

[24] J. P. T. Higgins and S. G. Thompson, "Quantifying heterogeneity in a meta-analysis," Statistics in medicine, vol. 21, no. 11, pp. 1539-1558, 2002.

[25] M. Egger, G. D. Smith, M. Schneider, and C. Minder, "Bias in meta-analysis detected by a simple, graphical test," $B M J$, vol. 315, no. 7109, pp. 629-634, 1997.

[26] G. Meiloud, I. Arfa, R. Kefi et al., "Type 2 diabetes in Mauritania: prevalence of the undiagnosed diabetes, influence of family history and maternal effect," Primary care diabetes, vol. 7, no. 1, pp. 19-24, 2013.

[27] R. Erasmus, E. Blanco Blanco, A. B. Okesina, J. Mesa Arana, Z. Gqweta, and T. Matsha, "Importance of family history in type 2 black South African diabetic patients," Postgraduate medical journal, vol. 77, no. 907, pp. 323-325, 2001.

[28] J.-C. Mbanya, L. N. Pani, D. N. Mbanya, E. Sobngwi, and J. Ngogang, "Reduced insulin secretion in offspring of African type 2 diabetic parents," Diabetes Care, vol. 23, no. 12, pp. 1761-1765, 2000.

[29] A. Amoah, S. Owusu, O. Ayittey, D. Schuster, and K. Osei, "Minimal model analyses of beta cell secretion, insulin sensitivity and glucose effectiveness in glucose tolerant, nondiabetic first-degree relatives of Ghanaian patients with type 2 diabetes and healthy control subjects," Ethnicity \& disease, vol. 11, no. 2, pp. 201-210, 2001. 
[30] C. N. Rotimi, G. Chen, A. A. Adeyemo et al., "A genome-wide search for type 2 diabetes susceptibility genes in West Africans: the Africa America Diabetes Mellitus (AADM) Study," Diabetes, vol. 53, no. 3, pp. 838-841, 2004.

[31] G. Chen, A. Adeyemo, J. Zhou et al., "Genome-wide search for susceptibility genes to type 2 diabetes in West Africans: potential role of C-peptide," Diabetes research and clinical practice., vol. 78, no. 3, pp. e1-e6, 2007.

[32] H. Zouali, E. H. Hani, A. Philippi et al., "A susceptibility locus for early-onset non-insulin dependent (type 2) diabetes mellitus maps to chromosome 20q, proximal to the phosphoenolpyruvate carboxykinase gene," Human molecular genetics., vol. 6, no. 9, pp. 1401-1408, 1997.

[33] L. Ji, M. Malecki, J. H. Warram, Y. Yang, S. S. Rich, and A. S. Krolewski, "New susceptibility locus for NIDDM is localized to human chromosome 20q," Diabetes, vol. 46, no. 5, pp. 876-881, 1997.

[34] K. Yamagata, N. Oda, P. J. Kaisaki et al., "Mutations in the hepatocyte nuclear factor- $1 \alpha$ gene in maturity-onset diabetes of the young (MODY3)," Nature, vol. 384, no. 6608, pp. 455-458, 1996.

[35] A. A. Adeyemo, F. Tekola-Ayele, A. P. Doumatey et al., "Evaluation of genome wide association study associated type 2 diabetes susceptibility loci in sub Saharan Africans," Frontiers in genetics, vol. 6, p. 335, 2015.

[36] A. A. Adeyemo, N. A. Zaghloul, G. Chen et al., “_ZRANB3_is an African-specific type 2 diabetes locus associated with betacell mass and insulin response," Nature Communications, vol. 10, no. 1, pp. 1-12, 2019.

[37] J. Chen, M. Sun, A. Adeyemo et al., "Genome-wide association study of type 2 diabetes in Africa," Diabetologia, vol. 62, no. 7, pp. 1204-1211, 2019.

[38] G. M. Bantie, A. A. Wondaye, E. B. Arike et al., "Prevalence of undiagnosed diabetes mellitus and associated factors among adult residents of Bahir Dar city, northwest Ethiopia: a community-based cross-sectional study," BMJ open, vol. 9, no. 10, p. e030158, 2019.

[39] T. Zenebe, H. Merga, and E. Habte, "A community-based cross-sectional study of magnitude of dysglycemia and associated factors in Southwest Ethiopia," International Journal of Diabetes in Developing Countries, vol. 39, no. 4, pp. 749-755, 2019.

[40] B. O. Bello-Ovosi, S. Asuke, S. O. Abdulrahman et al., "Prevalence and correlates of hypertension and diabetes mellitus in an urban community in North-Western Nigeria," Pan African Medical Journal., vol. 29, no. 1, pp. 1-7, 2018.

[41] A. T. Wondemagegn, H. M. Bizuayehu, D. D. Abie, G. M. Ayalneh, T. Y. Tiruye, and M. T. Tessema, "Undiagnosed diabetes mellitus and related factors in East Gojjam (NW Ethiopia) in 2016: a community-based study," Journal of public health research, vol. 6, no. 1, 2017.

[42] T. Tesfaye, B. Shikur, T. Shimels, and N. Firdu, "Prevalence and factors associated with diabetes mellitus and impaired fasting glucose level among members of federal police commission residing in Addis Ababa, Ethiopia," BMC endocrine disorders, vol. 16, no. 1, p. 68, 2016.

[43] F. Vuvor, M. Steiner-Asiedu, M. Armar-Klemesu, and S. Armah, "Population-based study of diabetic mellitus prevalence and its associated factors in adult Ghanaians in the greater Accra region," International Journal of Diabetes in Developing Countries, vol. 31, no. 3, pp. 149-153, 2011.
[44] T. Millogo, B. W. Bicaba, J. K. Soubeiga, E. Dabiré, I. Médah, and S. Kouanda, "Diabetes and abnormal glucose regulation in the adult population of Burkina Faso: prevalence and predictors," BMC Public Health, vol. 18, no. 1, p. 350, 2018.

[45] S. M. Abebe, Y. Berhane, A. Worku, and A. Assefa, "Diabetes mellitus in North West Ethiopia: a community based study," BMC Public Health, vol. 14, no. 1, p. 97, 2014.

[46] R. W. Mayega, D. Guwatudde, F. Makumbi et al., "Diabetes and pre-diabetes among persons aged 35 to 60 years in eastern Uganda: prevalence and associated factors," PLoS One, vol. 8, no. 8, p. e72554, 2013.

[47] E. A. Nyenwe, O. J. Odia, A. E. Ihekwaba, A. Ojule, and S. Babatunde, "Type 2 diabetes in adult Nigerians: a study of its prevalence and risk factors in Port Harcourt, Nigeria," Diabetes research and clinical practice, vol. 62, no. 3, pp. 177-185, 2003.

[48] L. K. Frank, A. Heraclides, I. Danquah, G. Bedu-Addo, F. P. Mockenhaupt, and M. B. Schulze, "Measures of general and central obesity and risk of type 2 diabetes in a Ghanaian population," Tropical Medicine \& International Health, vol. 18, no. 2, pp. 141-151, 2013.

[49] I. Danquah, G. Bedu-Addo, K.-J. Terpe et al., "Diabetes mellitus type 2 in urban Ghana: characteristics and associated factors," BMC Public Health, vol. 12, no. 1, p. 210, 2012.

[50] M. P. Chege, "Risk factors for type 2 diabetes mellitus among patients attending a rural Kenyan hospital," African journal of primary health care \& family medicine, vol. 2, no. 1, 2010.

[51] G. A. Engwa, F. N. Nwalo, C. C. Chikezie et al., "Possible association between ABCC8 C49620T polymorphism and type 2 diabetes in a Nigerian population," BMC Medical Genetics, vol. 19, no. 1, p. 78, 2018.

[52] I. K. Quaye, G. Ababio, and A. G. Amoah, "Haptoglobin 2-2 phenotype is a risk factor for type 2 diabetes in Ghana," Journal of atherosclerosis and thrombosis, vol. 13, no. 2, pp. 90-94, 2006.

[53] T. Chikowore, K. R. Conradie, G. W. Towers, and T. van Zyl, "Common variants associated with type 2 diabetes in a black South African population of Setswana descent: African populations diverge," Omics: a journal of integrative biology, vol. 19, no. 10, pp. 617-626, 2015.

[54] G. A. Engwa, F. N. Nwalo, C. E. Obi, C. Onyia, O. O. O. Wilfred Fon Mbacham, and B. Ewa Ubi, "Predominance of the a Allele but no Association of the KCNJ11 rs5219 E23K Polymorphism with Type 2 Diabetes in a Nigerian Population," Genetics \& Molecular Research, vol. 17, no. 1, 2018.

[55] I. Abdelhamid, K. Lasram, G. Meiloud et al., "E23K variant in _KCNJ11_ gene is associated with susceptibility to type 2 diabetes in the Mauritanian population," Primary Care Diabetes, vol. 8, no. 2, pp. 171-175, 2014.

[56] Y. Y. Yako, J. H. Madubedube, A. P. Kengne, R. T. Erasmus, T. S. Pillay, and T. E. Matsha, "Contribution of ENPP1, TCF7L2, and FTO polymorphisms to type 2 diabetes in mixed ancestry ethnic population of South Africa," African health sciences, vol. 15, no. 4, pp. 1149-1160, 2015.

[57] A. Olckers, G. W. Towers, A. van der Merwe, P. E. H. Schwarz, P. Rheeder, and A. E. Schutte, "Protective effect against type 2 diabetes mellitus identified within the _ACDC_gene in a black South African diabetic cohort," Metabolism, vol. 56, no. 5, pp. 587-592, 2007.

[58] B. Ayelign, M. Genetu, T. Wondmagegn, G. Adane, M. Negash, and N. Berhane, "TNF- $\alpha(-308)$ gene polymorphism and type 2 
diabetes mellitus in Ethiopian diabetes Patients," Diabetes Metabolic Syndrome and Obesity-Targets and Therapy, vol. Volume 12, pp. 2453-2459, 2019.

[59] I. Danquah, T. Othmer, L. K. Frank, G. Bedu-Addo, M. B. Schulze, and F. P. Mockenhaupt, "The TCF7L2 rs 7903146 (T) allele is associated with type 2 diabetes in urban Ghana: a hospital-based case-control study," BMC Medical Genetics, vol. 14, no. 1, p. 96, 2013.

[60] D. Nanfa, E. Sobngwi, B. Atogho-Tiedeu et al., "Association between the TCF7L2 rs12255372 (G/T) gene polymorphism and type 2 diabetes mellitus in a Cameroonian population: a pilot study," Clinical and translational medicine, vol. 4, no. 1, p. 17, 2015.

[61] M. Guewo-Fokeng, E. Sobngwi, B. Atogho-Tiedeu et al., "Contribution of the TCF7L2 rs7903146 (C/T) gene polymorphism to the susceptibility to type 2 diabetes mellitus in Cameroon," Journal of Diabetes \& Metabolic Disorders, vol. 14, no. 1, p. 26, 2015.

[62] A. Helgason, S. Pálsson, G. Thorleifsson et al., "Refining the impact of _TCF7L2_ gene variants on type 2 diabetes and adaptive evolution," Nature genetics, vol. 39, no. 2, pp. 218225, 2007.

[63] J. B. Meigs, L. A. Cupples, and P. W. Wilson, "Parental transmission of type 2 diabetes: the Framingham Offspring Study," Diabetes, vol. 49, no. 12, pp. 2201-2207, 2000.

[64] P. Poulsen, K. Ohm Kyvik, A. Vaag, and H. Beck-Nielsen, "Heritability of type II (non-insulin-dependent) diabetes mellitus and abnormal glucose tolerance-a population-based twin study," Diabetologia, vol. 42, no. 2, pp. 139-145, 1999.

[65] J. C. Florez, J. Hirschhorn, and D. Altshuler, "The inherited basis of diabetes mellitus: implications for the genetic analysis of complex traits," Annual review of genomics and human genetics, vol. 4, no. 1, pp. 257-291, 2003.

[66] J. C. Alcolado and R. Alcolado, "Importance of maternal history of non-insulin dependent diabetic patients," $B M J$, vol. 302, no. 6786, pp. 1178-1180, 1991.

[67] S. N. T. De Silva, N. Weerasuriya, N. M. W. De Alwis, M. W. A. De Silva, and D. J. S. Fernando, "Excess maternal transmission and familial aggregation of type 2 diabetes in Sri Lanka," Diabetes research and clinical practice, vol. 58, no. 3, pp. 173-177, 2002.

[68] H. Benrahma, I. Arfa, M. Charif et al., "Maternal effect and familial aggregation in a type 2 diabetic Moroccan population," Journal of Community Health, vol. 36, no. 6, pp. 943948, 2011.

[69] M. Viswanathan, M. I. McCarthy, C. Snehalatha, G. A. Hitman, and A. Ramachandran, "Familial aggregation of type 2 (non-insulin-dependent) diabetes mellitus in South India; absence of excess maternal transmission," Diabetic medicine, vol. 13, no. 3, pp. 232-237, 1996.

[70] B. D. Mitchell, C. M. Kammerer, L. J. Reinhart, M. P. Stern, and J. W. MacCluer, "Is there an excess in maternal transmission of NIDDM?," Diabetologia, vol. 38, no. 3, pp. 314-317, 1995.

[71] A. E. Harding, R. K. Petty, and J. A. Morgan-Hughes, "Mitochondrial myopathy: a genetic study of 71 cases," Journal of medical genetics, vol. 25, no. 8, pp. 528-535, 1988.

[72] D. J. Pettitt, K. A. Aleck, H. R. Baird, M. J. Carraher, P. H. Bennett, and W. C. Knowler, "Congenital susceptibility to NIDDM: role of intrauterine environment," Diabetes, vol. 37 , no. 5, pp. 622-628, 1988.
[73] D. Kibirige, W. Lumu, A. G. Jones, L. Smeeth, A. T. Hattersley, and M. J. Nyirenda, "Understanding the manifestation of diabetes in sub Saharan Africa to inform therapeutic approaches and preventive strategies: a narrative review," Clinical Diabetes and Endocrinology, vol. 5, no. 1, p. 2, 2019.

[74] J. van Tilburg, T. W. van Haeften, P. Pearson, and C. Wijmenga, "Defining the genetic contribution of type 2 diabetes mellitus," Journal of Medical Genetics, vol. 38, no. 9, pp. 569-578, 2001.

[75] M. C. Y. Ng, D. Shriner, B. H. Chen et al., "Meta-analysis of genome-wide association studies in African Americans provides insights into the genetic architecture of type 2 diabetes," PLoS genetics, vol. 10, no. 8, p. e1004517, 2014.

[76] Y. S. Cho, D. I. A. G. R. A. M. Consortium, C.-H. Chen et al., "Meta-analysis of genome-wide association studies identifies eight new loci for type 2 diabetes in east Asians," Nature genetics, vol. 44, no. 1, pp. 67-72, 2012.

[77] Y. Y. Yako, M. Guewo-Fokeng, E. V. Balti et al., "Genetic risk of type 2 diabetes in populations of the African continent: a systematic review and meta-analyses," Diabetes research and clinical practice, vol. 114, pp. 136-150, 2016.

[78] J. P. A. Ioannidis and T. A. Trikalinos, "Early extreme contradictory estimates may appear in published research: the Proteus phenomenon in molecular genetics research and randomized trials," Journal of clinical epidemiology, vol. 58, no. 6 , pp. 543-549, 2005.

[79] F. Tekola-Ayele, A. A. Adeyemo, and C. N. Rotimi, "Genetic epidemiology of type 2 diabetes and cardiovascular diseases in Africa," Progress in cardiovascular diseases, vol. 56, no. 3, pp. 251-260, 2013.

[80] D. L. Nicolae, E. Gamazon, W. Zhang, S. Duan, M. E. Dolan, and N. J. Cox, "Trait-associated SNPs are more likely to be eQTLs: annotation to enhance discovery from GWAS," PLoS genetics, vol. 6, no. 4, p. e1000888, 2010.

[81] T. E. Matsha, C. Pheiffer, S. E. Humphries, J. Gamieldien, R. T. Erasmus, and A. P. Kengne, "Genome-wide DNA methylation in mixed ancestry individuals with diabetes and prediabetes from South Africa," International Journal of Endocrinology, vol. 2016, 11 pages, 2016.

[82] K. A. C. Meeks, P. Henneman, A. Venema et al., "Epigenomewide association study in whole blood on type 2 diabetes among sub-Saharan African individuals: findings from the RODAM study," International journal of epidemiology, vol. 48, no. 1, pp. 58-70, 2018.

[83] E. Hall, P. Volkov, T. Dayeh et al., "Effects of palmitate on genome-wide mRNA expression and DNA methylation patterns in human pancreatic islets," BMC medicine., vol. 12, no. 1, p. 103, 2014. 